\title{
Ultrahigh Intensity Laser for Laser Wakefield Acceleration
}

\author{
Gerard Mourou, John Nees, and Subrat Biswal \\ Center for Ultrafast Optical Science, University of Michigan \\ 2200 Bonisteel Blvd, IST 1006, Ann Arbor, Michigan 48109-2099
}

\begin{abstract}
The next generation of high peak power CPA laser systems will be improved in compactness, simplicity, and cost. Yb:glass is a suitable choice for the amplifier medium in such a laser system necessary for an all-optical $\mathrm{GeV}$ accelerator.
\end{abstract}

The concept of Chirped Pulse Amplification (CPA) has revolutionized the generation of high peak power pulses as shown in Fig. 1 and made possible the generation of optical pulses of several terawatts by table top systems that are $10^{3}-10^{4}$ above what was obtained by the same size system $(1,2)$. The concept is very general and has been applied from the microjoule to the kilojoule using amplifying systems as diverse in size as doped fibers or building size amplifier built in national laser fusion facility like Lawrence Livermore National Laboratory, CEA, Osaka, etc... Although these recent achievements have been very impressive, there is still much room for improvement. The next generation of CPA laser systems will have vastly improved characteristics in terms of peak power near petawatt, compactness, simplicity and cost. These improvements will come from a more judicious choice of materials and pumping sources.

Right now one of the most important applications of CPA has been in the area of wakefield acceleration, a concept proposed in 1979 by Tajima and Dawson (3). Their concept has been recently verified by the important work of Clayton et al. (4), Umstadter et al. (5), Downer et al. (6), and Amiranoff et al. (7). For this particular application it is important that we get the peak intensity in the $10^{18} \mathrm{~W} / \mathrm{cm}^{2}$ range. In order to get the best efficiency between the laser and the accelerated electrons, it has been proposed to excite the plasma with a succession of pulses with a changing, pulse duration and interval between pulses. This concept is known as the RLPA (8) for Resonantly Driven Plasma Accelerator. Here, CPA is also ideal to produce a pulse with a prescribed time structure that will optimize the energy transfer between the laser pulse. The pulse duration is one of the most fundamental parameter. For single pulse excitation, (not RLPA), the optimum duration is between $50-100 \mathrm{fs}$. The focused pulse will create a plasma wave with a period about equal to the optical pulse duration. Associated with this plasma wave will be a very large field 


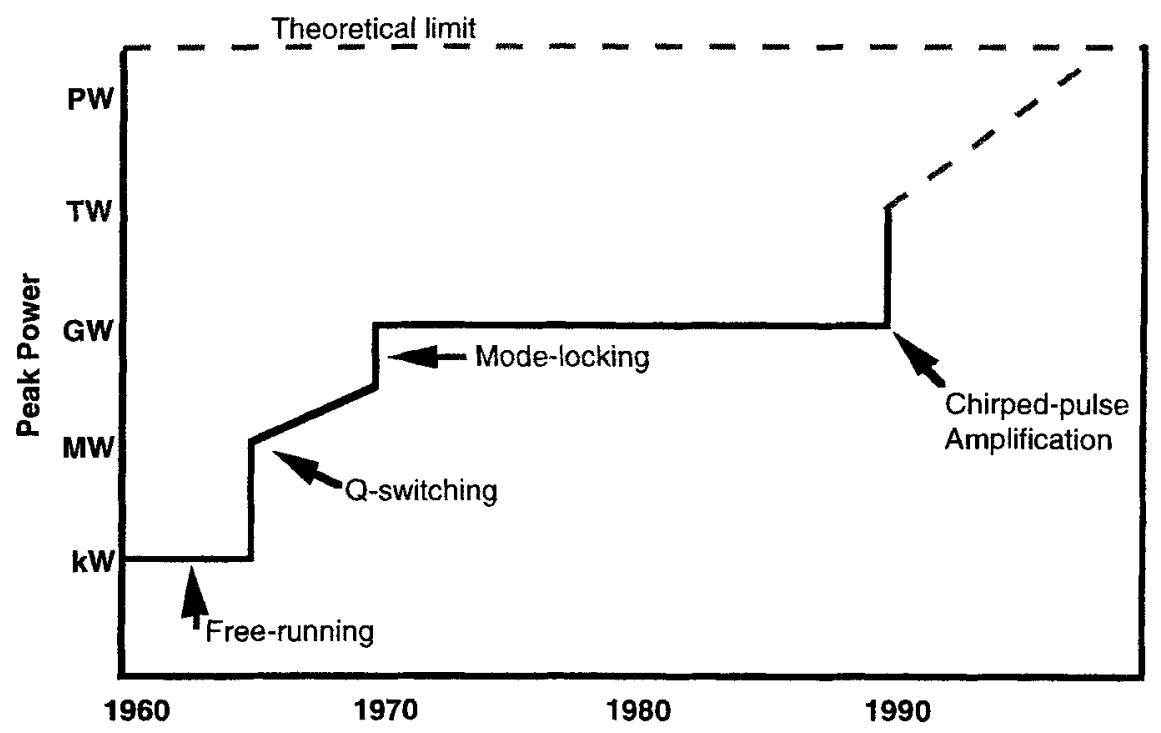

Figure 1. Evolution of laser peak power.

gradient of the order of $100 \mathrm{GeV} / \mathrm{m}$ that will form an acceleration bucket of about $50 \mathrm{fs}$ in duration. The very large field existing in the acceleration buckets is at the expense of their short duration. In order to have no energy spread we need to inject the electrons at one particular point of the acceleration bucket. This requirement becomes impossible to fill with present technology like, photogun injection where the shortest pulse with a significant amount of charge (of the order of one $\mathrm{nC}$ ) is of the order of $1 \mathrm{ps}$, that is 20 times larger than the bucket duration. This apparent insurmountable difficulty was solved by Umstadter et al. (5) that proposed that the injection could be done with a femtosecond auxiliary pulse, synchronized with the main pulse that would kick the electrons by ponderomotive force into the acceleration bucket. Because of the formidable gradients, the simulations show that a femtosecond low energy spread electron pulse with a charge of several $\mathrm{nC}$ could be very simply produced. This concept has been dubbed LILAC for Laser Injected Laser Accelerator. The laser wakefield combined with the LILAC opened the possibility to make an all optical $\mathrm{GeV}$ electron accelerators.

We are going to describe some of the CPA embodiments well suited in terms of high peak and average power, compactness, simplicity and efficiency for an All Optical GeV Accelerator. 


\section{CPA Laser for Wakefield Acceleration.}

The size of a CPA system scales with the saturation fluence $F_{s}=h v / \sigma$. The saturation fluence is inversely proportional to the cross section $\sigma$. The cross section of different laser materials can vary over many orders of magnitude. What defines a good energy storage materials is its ability to store optical energy before it is depleted by amplified spontaneous emission (ASE). The ASE increases exponentially with the product $\sigma \mathrm{Nl}$, where $\sigma$ is the stimulated emission cross section, $\mathrm{N}$ is the atom or molecule density, and 1 is the amplifier length. We see that for a given $\sigma \mathrm{Nl}$, a good storage medium will have the smallest emission cross section so the largest amount of atoms or molecules can be present in the smallest volume keeping the ASE to a low level.

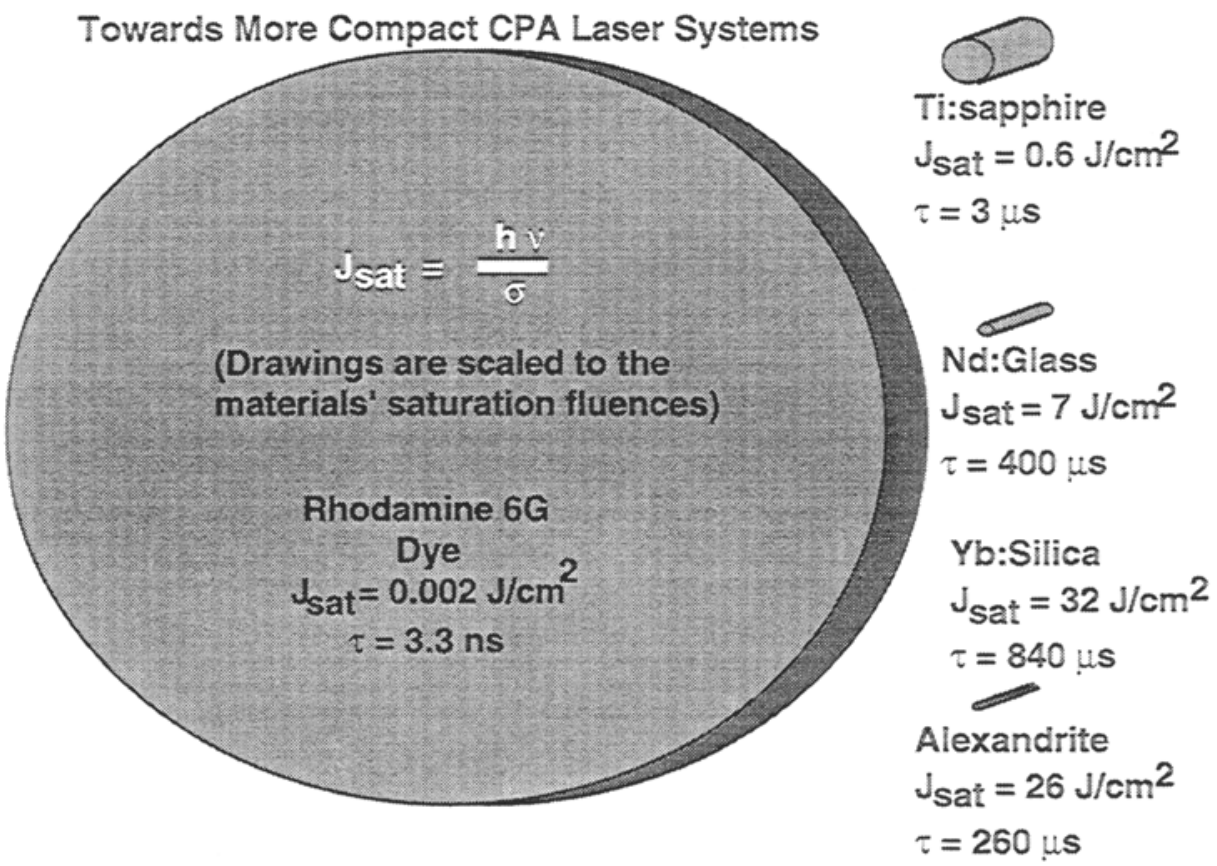

Figure 2. Relative area of various amplifier media for a given amount of energy

The lasing emission cross section can vary widely for different amplifying media from $10^{-16} \mathrm{~cm}^{2}$ for dye to $10^{-20} \mathrm{~cm}^{2}$ for Yb:glass, that is over 4 orders of magnitude. The fundamental problem which the CPA technique solved was the ability to extract a large amount of energy while keeping the nonlinear effects at a manageable level. This was demonstrated 10 years ago by stretching the pulse in time before the amplification. ${ }^{1}$ After energy extraction the pulse is compressed back to its 
initial value. Before this technique, only dye amplifier was used in a straight amplification configuration. With CPA, low emission cross section materials, that is good energy storage solid-state materials, can be used such as Ti:sapphire, Nd:glass, Cr:LiSAF, etc... Fig. 2 shows the relative physical cross sectional area of different amplifying media for a given amount of stored energy. It clearly expresses the vast difference between the different materials. Of course all these materials have a broad gain bandwidth, necessary to amplify ultrashort pulses in the subpicosecond domain.

Besides the good energy storage characteristics, one additional property that is particularly important is the storage time $\tau_{s}$. This characteristic can also vary widely over many orders of magnitude, between the different amplifier materials. For instance, dyes have storage time in the nanoseconds, while for Ti:sapphire it is $3 \mu \mathrm{s}$. For Nd:glasses it is $300 \mu \mathrm{s}$ and up to $2 \mathrm{~ms}$ for $\mathrm{Yb}$ :glass. This time imposes a very different constraint on the pump power for different materials. If we want to store one joule into an amplifier, the pump power has to be in the gigawatts for the dye, megawatt for Ti:sapphire, and kilowatt for Nd:glass and Yb:glass. The size, cost, and complexity of the pump source will here again be a very important function of the pump peak power.

Laser acceleration will need high peak power, but also high average power. Since the inception of CPA, the average power of femtosecond systems has increased by at least two orders of magnitude. Fig. 3 shows the average power for different repetition rate femtosecond CPA systems. Average power is a function of the quantum defect between the absorption and emission wavelengths and the thermal conductivity of the amplifying media. The average power can be enhanced by rotating or translating the amplifier (10), that would be made easier if the physical dimensions of the amplifier are small and not birefringent.

Over the past few year at the University of Michigan we looked at alternatives to enhance the compactness and simplicity of CPA systems by using a judicious combination of amplifier materials and pump sources. Our search has been centered on long storage time materials with low cross section (good energy storage) that could be pumped with an inexpensive pump source working in free running mode or with laser diodes. Our first choice has been the combination of Nd:glass and free running alexandrite (11). This had the advantage to use an inexpensive material, Nd:glass, with excellent storage energy characteristics due to the low cross section corresponding to a stored energy density greater than 1 $\mathrm{J} / \mathrm{cm}^{3}$. A free running alexandrite laser is an inexpensive tunable source of photons and can deliver of the order of $5 \mathrm{~J}$ at $10 \mathrm{~Hz}$. Its tunability over the absorption bandwidth of Nd:glass between 700-800 nm allows for the adjustment of the absorption length. Also the free running pump pulse duration time is of the order of $100 \mu \mathrm{s}$, which is a fraction of the Nd:glass storage time of $300 \mu \mathrm{s}$. The long pump pulse seriously reduces the 
damage threshold problem in the optical components and permits pumping of the Nd:glass with a high energy fluence on the order of 100 $\mathrm{J} / \mathrm{cm}^{2}$. Recently an alexandrite-pumped Nd:glass $10 \mathrm{~Hz}, 50 \mathrm{~mJ}$ system with bandwidth to support a sub-picosecond pulse was demonstrated (12). The pulse duration due to the relatively narrow gain bandwidth of $\mathrm{Nd}$ :silicate of $25 \mathrm{~nm}$ was of the order of $400 \mathrm{fs}$ after compression. We have also shown that this pulse duration could be further reduced to the sub$100 \mathrm{fs}$ range, by frequency doubling the compressed pulse using a scheme based on two KDP crystals, one for predelay and one for harmonic generation (13). The conversion efficiency was $75 \%$. The pulse duration was measured to be only of the order of $100 \mathrm{fs}$. We think that the observed temporal broadening was due to nonlinear effects in the crystal due small scale self focusing.

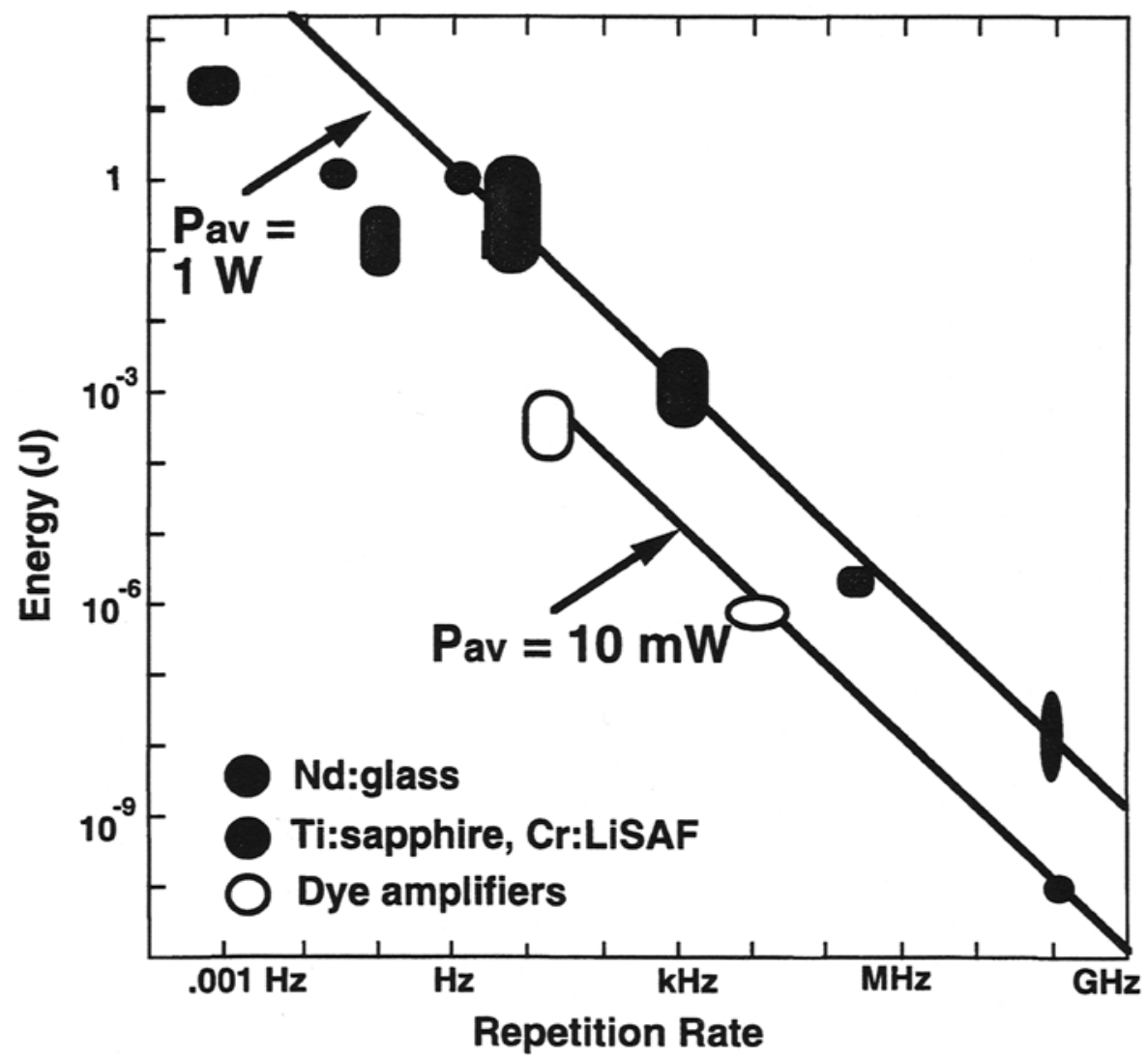

Figure 3. Average power for various high peak power laser systems.

Lately our efforts have concentrated on $\mathrm{Yb}$ : glass pumped with a free running flashlamp pumped Ti:sapphire and Cr:LiSAF. As we will see later, Yb:glass is ideal for diode pumping. Yb:glass has a very large 
bandwidth to accommodate $30 \mathrm{fs}$ pulse duration $(14,15)$ and a very good storage efficiency of the order of $10 \mathrm{~J} / \mathrm{cm}^{3}$. Also, the upper level lifetime of $\mathrm{Yb}$ :glass of up to $2 \mathrm{~ms}$, makes this material ideal for laser diode pumping. The simple two electronic energy level structure of $\mathrm{Yb}^{3+}$ allows for a high doping concentration of $>10^{21} / \mathrm{cm}^{3}$ in glass. If all the ions could be inverted and no ASE was present, the $10^{21} / \mathrm{cm}^{3}$ concentration corresponds to a stored energy density of $200 \mathrm{~J} / \mathrm{cm}^{3}$. The small quantum defect between the absorption band at $900-980 \mathrm{~nm}$ and emission at $1000-1030$ $\mathrm{nm}$ is good for high average power operation. Free running Ti:sapphire and Cr:LiSAF are excellent laser diode simulators, but are also rugged, cheap and compact source of photons. For instance with a quarter inch diameter Cr:LiSAF rod, a free-running, multi-mode output of $10 \mathrm{~J}$ at 900 $\mathrm{nm}$ can be obtained.

In order to extract efficiently the energy from the amplifier, one technique is to have an input fluence $F_{\text {in }}$ of the order of the saturation fluence of the material $F_{s}=h v / \sigma$. For superior energy storage materials, $F_{s}$ is very large in the range of $5 \mathrm{~J} / \mathrm{cm}^{2}$ for Nd:glass to $50 \mathrm{~J} / \mathrm{cm}^{2}$ for $\mathrm{Yb}$ :glass. This saturation fluence corresponds to the bulk damage threshold value for the input stretched pulse duration of a few nanoseconds. The solution to the problem of damage threshold will be a combination of large pulse stretching, to several nanoseconds - it has been determined empirically that the damage threshold of dielectrics increases with the square root of the pulse duration - and techniques to enhance damage thresholds. Superpolishing techniques can significantly enhance the damage threshold and rely on chemical etching, ion-beam polishing, or laser conditioning (16). Of course because of their large saturation fluences, the amplifier physical cross section will be small. For a $1 \mathrm{~J}$ system, the physical cross section will be of the order of one millimeter so the damage conditioning has to be only done over a small area of $1 \mathrm{~mm}$. Another solution to extract the energy is to use a low gain regenerative amplifier in which the population inversion is gradually depleted resulting in a peak fluence below the saturation fluence (17).

\section{Theoretical Peak Power and Rabi Intensity.}

The highest peak power can be estimated, by recognizing that the maximum extractable energy from an optical amplifier is dictated by the saturation fluence Fs=hv/ $\sigma$. This energy, can be extracted by a pulse, limited by the gain bandwidth $\Delta \omega$ that will ultimately limit the pulse duration to approximately $1 / \Delta \omega$. We end up with a very simple expression for the theoretical peak power $\mathrm{P}_{\mathrm{th}}=\mathrm{hv} \Delta \omega / \sigma$. Note, that of course this is the maximum extractable power per unit area.

It is easy to recognize that this power corresponds in fact to the Rabi intensity necessary to take the excited population to the ground state in a 
medium. The theoretical peak powers for the most commonly used CPA materials are listed in table 1.

Table 1. Theoretical peak power per $\mathrm{cm}^{2}$ of different laser materials.

$\begin{array}{lccccc}\begin{array}{l}\text { Laser } \\ \text { material }\end{array} & \begin{array}{c}\sigma \\ \left(10^{-20} \mathrm{~cm}^{2}\right)\end{array} & \begin{array}{c}\Delta \lambda \\ (\mathrm{nm})\end{array} & \begin{array}{c}\Delta t \\ (\mathrm{fs})\end{array} & \begin{array}{c}\tau_{\mathrm{s}} \\ (\mu \mathrm{s})\end{array} & \begin{array}{c}\mathrm{P}_{\text {th }} \\ \left(\mathrm{TW} / \mathrm{cm}^{2}\right)\end{array} \\ \begin{array}{l}\text { Ti:sapphire } \\ \text { Nd:silicate }\end{array} & 40 & 200 & 5 & 3 & 130 \\ \text { Cr:LiSAF } & 2.4 & 30 & 60 & 400 & 140 \\ \text { Alexandrite } & 5 & 150 & 7 & 70 & 650 \\ \text { Yb:silicate } & 1 & 100 & 10 & 250 & 2500 \\ & 0.5 & 100 & 15 & 800 & 2500\end{array}$

\section{Size of CPA wake-field System.}

An All Optical GeV Accelerator using the RLPA and the LILAC concepts will require few terawatt peak power pulses with sub-100 fs duration. A quick look at the table 1 indicates that this peak power could be obtained by using a CPA based Yb:glass system with a $1 \mathrm{~mm}$ diameter. The fact the rod can be very small, opens the possibility for efficient cooling by rotating the glass rod for instance. Or by changing the rod for each shot. The advantage of glass here is that it is very cheap and can be grown to a large size. The alignment could be done for each shot using adaptive optic techniques at $10 \mathrm{~Hz}$ for instance.

\section{Conclusion.}

We have shown that CPA is entering a new phase where we will intentionally take advantage of the exceptional energy storage, long lifetime and laser diode pumping, of materials such as $\mathrm{Yb}$ :glass. Yb:glass can store more than $10 \mathrm{~J} / \mathrm{cm}^{3}$, with a long storage lifetime of $2 \mathrm{~ms}$, and can be easily and directly pumped with free running lasers like Ti:sapphire, Cr:LiSAF, or laser diodes. The obtainable pulse duration should be of the order of $30 \mathrm{fs}$. The system will be very compact with a $\mathrm{mm}$ diameter rod and could be ideally suited for producing pulses at the terawatt level that will be necessary to inject and accelerate electrons to the GeV level using the LILAC and the RLPA at a $10 \mathrm{~Hz}$ repetition rate. 


\section{Acknowledgments}

We gratefully thank M. Myers and S. Jiang of Kigre Inc. for their fruitful discussions and for providing the $\mathrm{QX} / \mathrm{Yb}$ glass samples. We also thank ELIGHT Laser Systems and Polytec for providing the flashlamp-pumped Ti:sapphire laser.

This research was partially supported by the National Science Foundation through the Center for Ultrafast Optical Science under STC PHY 8920108.

\section{References}

1. Strickland, D. and Mourou, G., Opt. Commun. 56, 219 (1985).

2. Perry, M. D. and Mourou, G., Science 264, 917-24 (1994).

3. Tajima, T. and Dawson, J. M., Phys. Rev. Lett. 43, 267 (1979).

4. Modena, A., et al. Nature 377, 606 (1995).

5. Umstadter, D., Chen, S. Y., Maksimchuk, A, Mourou, G., and Wagner, R., Science 273, 472 (1996).

6. Downer, M., et al. Bullet. Amer. Phys. Soc. 40, 1892 (1995).

7. Amiranoff, F., et al. Adv. Accel. Conc., AIP Conf. Proc. 335, 612-34 (1995).

8. Umstadter, D., Esarey, E., and Kim, J. Phys. Rev. Lett. 72, 1224 (1994).

9. Nakajima, K., et al. Phys. Rev. Lett. 74, 4428 (1995).

10. Biswal, S., Nees, J., and Mourou, G., submitted to Applied Opt.

11. Squier, J., Coe, S., Clay, K., and Mourou, G., Opt. Commun. 92, 73 (1992).

12. Biswal, S., Coe, J. S., and Mourou, G., "High repetition rate, subpicosecond alexandrite-pumped $\mathrm{Nd}$ :glass amplifier system," presented at the Conference on Lasers and Electro-Optics, Baltimore, MD, May 1995.

13. Chien, C. Y., Korn, G., Coe, J. S., Squier, J., Mourou, G., and Craxton, R. S., Optics Letters 20, 353 (1995).

14. Walton, D., Nees, J., and Mourou, G., Opt. Lett. 21, 1061 (1996).

15. Pask, H. M., Carman, R. J., Hanna, D. C., Tropper, A. C., Mackechnie, C. J., Barber, P. R., and Dawes, J. M., IEEE JQE 1, 2-13 (1995).

16. Koechner, W., Solid-State Laser Engineering, Berlin: Springer-Verlag, 1992, ch. 11, pp. 579-89.

17. Biswal, S., Druon, F., Nishimura, A., Nees, J., and Mourou, G., submitted to the Conference on Lasers and Electro-Optics, Baltimore, MD (1997). 